Article

\title{
In Vitro Activity of 30 Essential Oils against Bovine Clinical Isolates of Prototheca zopfii and Prototheca blaschkeae
}

\author{
Simona Nardoni ${ }^{1, *}$, Francesca Pisseri ${ }^{2}$, Luisa Pistelli ${ }^{3}$, Basma Najar ${ }^{3}$, Mario Luini ${ }^{4}$ ([) and \\ Francesca Mancianti ${ }^{1}$ \\ 1 Dipartimento di Scienze Veterinarie, Università di Pisa, 56124 Pisa PI, Italy; francesca.mancianti@unipi.it \\ 2 Scuola Cimi Roma, 00153 Roma RM, Italy; info@francescapisseri.it \\ 3 Dipartimento di Farmacia, Università di Pisa, 56126 Pisa PI, Italy; luisa.pistelli@unipi.it (L.P.); \\ basmanajar@hotmail.fr (B.N.) \\ 4 Istituto Zooprofilattico Sperimentale della Lombardia e dell'Emilia, Sezione di Lodi, 26900 Lodi LO, Italy; \\ mariovittorio.luini@izsler.it \\ * Correspondence: simona.nardoni@unipi.it; Tel.: +39-050-221-6952
}

Received: 19 March 2018; Accepted: 18 April 2018; Published: 24 April 2018

\begin{abstract}
Protothecal mastitis poses an emergent animal health problem in dairy herds, with a high impact on dairy industries, causing heavy economic losses. Current methods of treating protothecal infections are ineffective, and no drug is licensed for use in cattle. The aim of the present study was to check the antialgal activity of 30 chemically defined essential oils (EOs) against Prototheca zopfii and Prototheca blaschkeae isolated from the milk of dairy cows with mastitis. A microdilution test was carried out to estimate the antialgal effectiveness of the selected chemically defined EOs. The microdilution test showed different degrees of inhibition among the examined Prototheca species. The activity of some of the examined EOs seem interesting. In particular, Citrus paradisi yielded the lowest minimal inhibitory concentration values $(0.75 \%)$ for both algal species. P. zopfii appeared to be more sensitive to EOs in comparison to P. blaschkeae. The present study investigated the in vitro susceptibility of P. zopfii and P. blaschkeae to a wide range of EOs, obtained from different botanical families. Further investigations are necessary to evaluate the efficacy of EO-based formulations intended for the disinfection of both udder and milking products.
\end{abstract}

Keywords: Prototheca zopfii; Prototheca blaschkeae; cattle; mastitis; treatment; essential oils; microdilution

\section{Introduction}

Prototheca spp. are achlorophyllous algae, strictly related to Chlorella. The genus includes both saprophytic (i.e., Prototheca stagnora and Prototheca ulmea) and pathogenic (i.e., Prototheca zopfii, Prototheca wickerhami, Prototheca blaschkeae, Prototheca cutis, and Prototheca miyajii) species [1-4]. Generally, human and animal protothecoses are considered as rare pathological conditions, apart from the case of bovine mastitis. Bovine protothecosis appears to prevail in contaminated environments where poor milking hygiene exists [5], and is enhanced by the biofilm-producing ability of $P$. zopfii, which may favor its persistence in milking and dairy environments $[6,7]$.

Protothecal mastitis poses an emergent problem in dairy herds [8], and is primarily caused by P. zopfii [9] and P. blaschkeae [10]. These algae induce severe granulomatous damage to bovine udders, leading to significant irreversible reductions in milk production [11]. This disease has a large negative impact on dairy industries, provoking heavy economic losses. The organoleptic characteristics of milk from infected cows are negatively affected, and thus, these animals should be removed from the herd. 
Furthermore, $P$. zopfii can be identified as a public health concern, as it is strongly related to increased mortality rates in immunocompromised human patients [12,13].

Current methods of treating protothecal infections are not effective, and P. zopfii in particular appears to be resistant to several antimycotic drugs [14,15]. Only amphotericin B appears promising, according to in vitro experiments [16].

Alternative treatments to the conventional antibiotics are welcome. Several natural products, including several plant extracts and essential oils (EOs), have already been examined. A methanolic extract of Clematis vitalba was found to be effective against both P. wickerhami and P. zopfii [17], while a leaf extract of Camellia sinensis was active against some strains of $P$. wickerhami, but ineffective against $P$. zopfii [18]. EOs from different botanical species have shown antimicrobial and antifungal actions [19], and can be used as natural preservatives in food [20], or for in vivo treatment of some fungal diseases [21] and prevention of bacterial infections [22]. EOs are multicomponent substances, and their antiseptic activity is often linked to their variety of components, and to their synergistic or antagonistic activity [23]. Furthermore, these natural compounds show anti-biofilm effects [24,25], which renders their use feasible for environmental hygiene.

Data about the sensitivity of Prototheca spp. to EOs are scant, and the effectiveness of these substances has only been determined against $P$. zopfii [26] in comparison to $P$. wickerhami [15]. In particular, EOs from Melaleuca alternifolia and Citrus bergamia [15], and from Cynnamomum zeylanicum and Thymus vulgaris [26] exerted significant in vitro activity. At the same time, EOs from Mentha piperita and Satureja hortensis appeared effective in significantly reducing clinical signs of inflammation and fibrosis in vivo, in a murine model of cutaneous protothecosis induced by $P$. zopfii [27].

The aim of the present study was to assess the antialgal activity of 30 chemically defined EOs against $P$. zopfii and P. blaschkeae isolated from the milk of dairy cows with mastitis.

\section{Materials and Methods}

EOs from Pimpinella anisum, Illicium verum, Santalum album, Helichrysum italicum, Rosmarinus officinalis, Lavandula hybrida, Pelargonium graveolens, Salvia sclarea, Cynnamomum zeylanicum, Foeniculum vulgare, Syzygium aromaticum, Boswellia sacra, Anthemis nobilis, Citrus paradisi, Citrus bergamia, Citrus aurantium, Citrus aurantium var. dulcis, Citrus limon, Cymbopogon citratus, Ocimum basilicum, Origanum majorana, Thymus vulgaris, Litsea cubeba, Origanum vulgare, Satureja montana, Cistus ladanifer, Picea abies, Anethum graveolens, Thymus capitatus, and Myrtus communis were used for the in vitro assays. All EOs were purchased from Flora s.r.l. (Lorenzana, Pisa, Italy) and were chemically defined. These oils were selected on the basis of the literature (C. bergamia, S. montana), or their antimycotic properties previously evaluated on different molds and yeasts (i.e., T. vulgaris, O. vulgare, L. cubeba), or their anti-inflammatory, lenitive (i.e., A. nobilis, H. italicum, L. hybrida, R. officinalis, S. sclarea), and immunostimulating activity (i.e., C. limon).

The gas chromatography-mass spectrometry (GC-MS) analysis was performed using a Varian CP-3800 gas chromatograph equipped with a DB-5 capillary column and a Varian Saturn 2000 ion trap mass detector (Varian Inc., Walnut Creek, CA, USA). Analytical conditions and identification of constituents were accomplished according to Pistelli et al. [28].

Statistical analysis was performed using the software package Past 3 (version 3.15). The hierarchical cluster analysis (HCA) was performed using Ward's method, with squared Euclidian distances as a measure of similarity.

P. zopfii and P. blaschkeae isolated from bovine mastitic milk were used for the in vitro tests. A single isolate for each species was assayed. The organisms were kindly provided by Biobank IZSLER (Brescia, Italy), were characterized by PCR/DNA resolution melting analysis [29], and were maintained onto Prototheca Isolation Medium (PIM) at $37^{\circ} \mathrm{C}$. A microdilution test was carried out to estimate the antialgal effectiveness of the selected EOs. The technique was performed using a broth microdilution assay, following the approved standard recommended for yeasts by Clinical and Laboratory Standards Institute $\mathrm{M}_{27 \mathrm{~A}_{3}}$ [30], finishing at a $4 \%$ dilution. This dilution was chosen as the highest concentration 
of active that can be administered intramammary [31]. Minimal inhibitory concentration (MIC) was calculated as the lowest dilution at which the algae failed to grow after running the assay in triplicate. Conventional, commonly employed anti-Prototheca drugs (i.e., amphotericin B, voriconazole, and posaconazole) were also used. In detail, the in vitro susceptibility of the two algal species was assessed by E-test [32].

\section{Results}

All the identified compounds in the 30 EOs are listed in Table 1, while the main class of constituents are reported in Table 2. The total identified volatile fractions ranged from $86.2 \%$ in S. sclarea to $100.0 \%$ in C. aurantium, C. bergamia, C. lemon, C. aurantium var. dulcis, I. verum, L. hybrida, and S. album. The composition of all the EOs revealed more than 290 compounds; however, only those with a percentage of $5 \%$ or more are reported in Table 1 . The main compounds were represented by monoterpene hydrocarbons and oxygenated monoterpenes, while oxygenated sesquiterpenes were abundant only in S. album (88.8\%). I. verum, P. abies, and S. album showed high percentages of phenylpropanoids (above 90\%) which are also the main class of compounds found in F. vulgare, although in a lower percentage (56.5\%). Non-terpenes were present in high amounts in A. nobilis (esters, $75.6 \%$ ) and C. zeylanicum (56.5\%).

Statistical analysis was used to assess the high volume of information provided by the EOs' composition. The classes of compounds in the EOs were subjected to multivariate analysis. Hierarchical cluster analysis HCA showed the presence of two main groups (Figure 1). The first group (A) includes the following oils: S. sclarea, T. capitatus, P. graveolens, A. graveolens, C. citratus, L. hybrida, H. italicum, O. basilicum, S. montana, T. vulgaris, O. majorana, L. cubeba, and O. vulgare. The second group (B) included all remaining oils, and it was further divided into two subgroups: B2 with EOs from C. zeylanicum, A. nobilis, S. album, S. aromaticum, P. anisum, I. verum, and F. vulgare, and B1 with the other EOs.

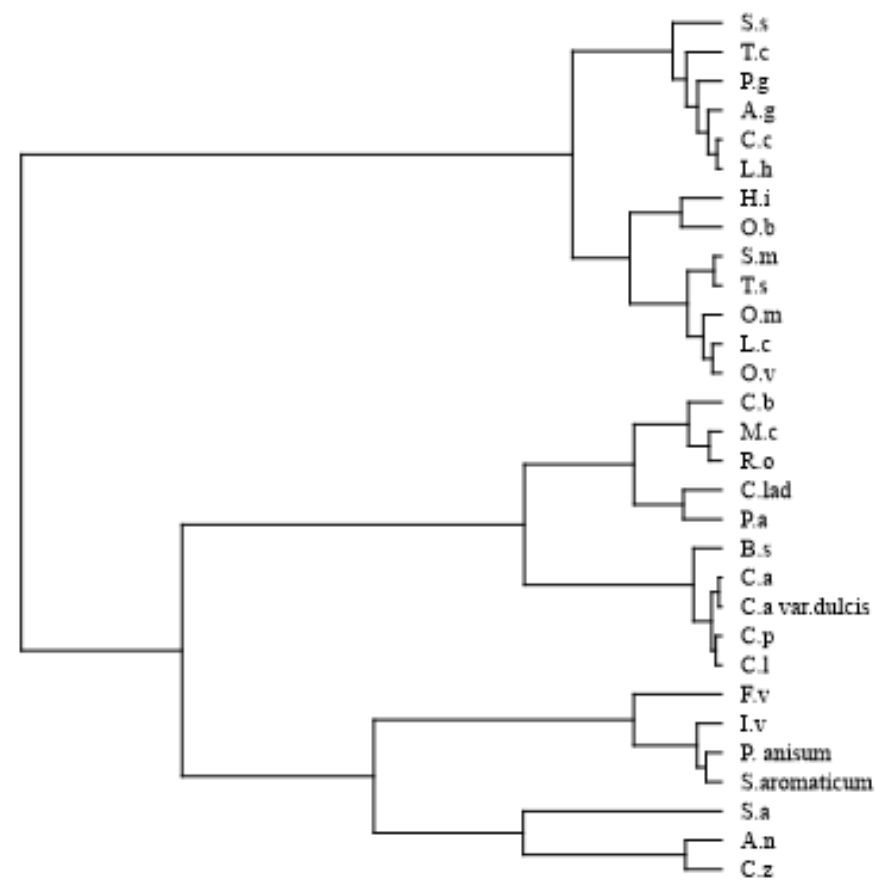

Figure 1. Dendrogram of the hierarchical cluster analysis (HCA) of the 30 tested essential oils (EOs).

Principal compound analysis (PCA), where the first axis (PC1) explained for $45.4 \%$ and the second axis (PC2) for $33.0 \%$ (which resumed $78.4 \%$ of the total variability, Figure 2), evidenced that oxygenated monoterpenes $(\mathrm{OMs})$ was the major class of compounds in all the EOs belonging to group A in HCA, and its percentages ranged from $40.6 \%$ in H. italicum EO to $89.2 \%$ in T. capitatus EO. The EOs that 
showed high amounts of phenylpropanoids were located in the upper-right quadrant of the PCA and referred to the plants present in subgroup B2 of the HCA, where P. anisum, I. verum, and S. aromaticum were characterized by the highest percentage of phenylpropanoids (up to 90\%), while C. zeylanicum and A. nobilis oils evidenced relevant amounts of non-terpene derivatives ( $57.9 \%$ and $75.6 \%$ respectively). S. album EO that belonged to the same subgroup B2 was characterized by the highest percentage of oxygenated sesquiterpenes $(88.8 \%)$. The presence of monoterpene hydrocarbons was predominant in the EOs of the plants inserted in subgroup B1, especially for all Citrus spp. (up to 90\%), except for C. bergamia, which was characterized by an equivalent amount of monoterpene hydrocarbons and oxygenated monoterpenes.

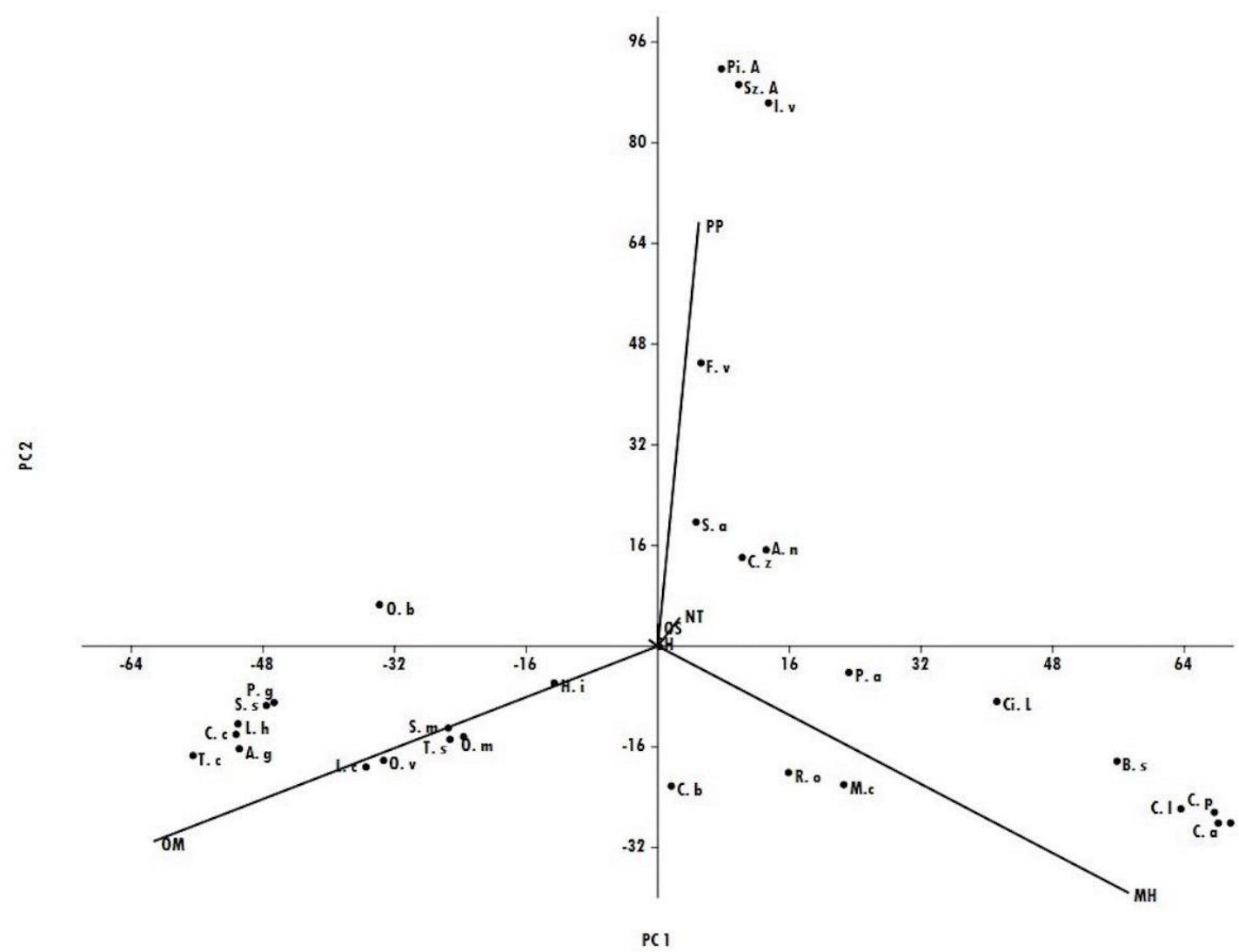

Figure 2. The compound analysis (PCA) plot of the main classes of compounds in the different EOs analyzed. 
Table 1. (a) Chemical composition of the first part of EOs tested (1-14); (b) Chemical composition of the second part of tested EOs (15-30).

(a)

\begin{tabular}{|c|c|c|c|c|c|c|c|c|c|c|c|c|c|c|c|c|}
\hline Compounds * & Class & LRI ${ }^{\S}$ & A.n & A.g & B.s & C.z & C. $a$ & C.b & C.l & C.p & C.a.dul & C.la & C.c & $F . v$ & H.i & I.v \\
\hline Propyl butanoate & EST & 898 & 5.5 & & & & & & & & & & & & & \\
\hline$\alpha$-Thujene & MH & 932 & & & 54.2 & 0.3 & & 0.3 & 0.4 & & & & 0.1 & & 7.2 & \\
\hline Tricyclene & MH & 938 & & & 0.2 & 1.4 & 0.4 & 1.1 & 1.9 & & 0.6 & & 0.2 & 8.6 & & 1.4 \\
\hline$\alpha$-Pinene & MH & 940 & 1.2 & 0.3 & 6.2 & & & & & 0.5 & & 45.0 & & & & \\
\hline$\alpha$-Fenchene & MH & 951 & & & & & & & & & & & & & 1.2 & \\
\hline Camphene & MH & 955 & 0.8 & & 0.8 & 0.6 & & & & & & 1.0 & 1.1 & 0.3 & & \\
\hline Thuja-2.4(10)-diene & MH & 959 & & & 7.3 & & & & & & & & & & & \\
\hline$\beta$-Pinene & MH & 981 & 0.2 & & 1.1 & 0.5 & 0.1 & 5.4 & 11.9 & 0.1 & & 0.6 & & 1.0 & 1.0 & \\
\hline$\alpha$-Terpinene & MH & 1019 & & & 5.1 & 1.0 & & & 0.2 & & & 0.1 & & & 0.1 & 0.2 \\
\hline$p$-Cymene & MH & 1028 & & 3.2 & & 3.0 & & 0.1 & 0.2 & & & 6.1 & & 1.9 & 1.1 & 0.1 \\
\hline Limonene & MH & 1032 & 0.7 & 3.2 & 0.4 & & 94.7 & 33.2 & 65.7 & 92.2 & 95.5 & 1.8 & 2.0 & 6.5 & 7.0 & 3.9 \\
\hline$\beta$-Phellandrene & $\mathrm{MH}$ & 1033 & & & & 5.9 & & & & & & & & & & \\
\hline 1.8-Cineole & $\mathrm{OM}$ & 1036 & & & & & & & & & & & 0.3 & & 2.3 & \\
\hline Isobutyl angelate & EST & 1053 & 34.5 & & & & & & & & & & & & & \\
\hline$\gamma$-Terpinene & MH & 1062 & & & 0.1 & & & 6.4 & 9.3 & & & & & 0.3 & 0.3 & 0.1 \\
\hline Artemisia ketone & $\mathrm{MH}$ & 1065 & 7.4 & & & & & & & & & & & & & \\
\hline Fenchone & $\mathrm{OM}$ & 1090 & & & & & & & & & & & & 20.1 & & \\
\hline trans-Sabinene hydrate & $\mathrm{OM}$ & 1101 & & & & & & & 0.2 & & & & & & & \\
\hline Linalool & $\mathrm{OM}$ & 1102 & & & 0.2 & 6.3 & 0.4 & 14.2 & & 0.2 & 0.5 & 0.5 & 1.5 & 0.4 & 0.8 & 0.2 \\
\hline Camphor & $\mathrm{OM}$ & 1148 & & & & & & & & & & & & 0.5 & & \\
\hline Isoamyl angelate & EST & 1162 & 18.7 & & & & & & & & & & & & & \\
\hline Propyl tiglate & EST & 1166 & 5.3 & & & & & & & & & & & & & \\
\hline 4-Terpineol & $\mathrm{OM}$ & 1180 & & & & 0.3 & & & & & & & & & 0.2 & 0.3 \\
\hline 3.9-Epoxy-p-menth-1-ene & $\mathrm{OM}$ & 1186 & & 12.0 & & & & & & & & & & & & \\
\hline Menthyl chavicol & PP & 1198 & & & & & & & & & & & & 8.6 & & 0.3 \\
\hline Citronellol & $\mathrm{OM}$ & 1231 & & & & & & & & & & & & & & \\
\hline Neral & $\mathrm{OM}$ & 1242 & 0.1 & & & & & 0.4 & 0.7 & & & & 35.2 & & & \\
\hline Carvone & $\mathrm{OM}$ & 1248 & & 70.4 & & & & & & & & & & & & \\
\hline Geraniol & $\mathrm{OM}$ & 1259 & & & & & & & & & & & 4.4 & & & \\
\hline Linalyl acetate & $\mathrm{OM}$ & 1260 & 0.5 & & & & 1.4 & 31.7 & & & & & & & & \\
\hline (E)-Cinnamaldehyde & NT & 1274 & & & & 56.4 & & & & & & & & & & \\
\hline Geranial & $\mathrm{OM}$ & 1276 & & & & & & 0.4 & 1.2 & & 0.1 & & 38.4 & & & \\
\hline Citronellyl formate & $\mathrm{OM}$ & 1280 & & & & & & & & & & & & & & \\
\hline iso-Bornyl acetate & $\mathrm{OM}$ & 1287 & & & & & & & & & & & & & & \\
\hline (E)-Anethol & PP & 1290 & & 0.3 & & & & & & & & & & 46.9 & & 89.8 \\
\hline Carvacrol & $\mathrm{OM}$ & 1301 & & & & & & & & & & & & & & \\
\hline Thymol & $\mathrm{OM}$ & 1307 & & & & & & & & & & 0.3 & & & & \\
\hline
\end{tabular}


Table 1. Cont.

\begin{tabular}{|c|c|c|c|c|c|c|c|c|c|c|c|c|c|c|c|c|}
\hline Compounds * & Class & LRI $^{\S}$ & A.n & A.g & B.s & C.z & C. $a$ & C.b & C.l & C.p & C.a.dul & C.la & C.c & $F . v$ & H.i & I.v \\
\hline$\alpha$-Limonene diepoxide & $\mathrm{OM}$ & 1347 & & & & & & & & & & & & & & \\
\hline Eugenol & PP & 1361 & & & & 3.0 & & & & & & & & & & \\
\hline Neryl acetate & $\mathrm{OM}$ & 1368 & & & & & & 0.8 & 0.7 & & & & & & 31.8 & \\
\hline$\beta$-Caryophyllene & $\mathrm{SH}$ & 1418 & & & & 10.3 & 0.2 & 0.7 & 0.4 & 0.7 & & 0.2 & 2.3 & & 3.1 & 0.3 \\
\hline Neryl propanoate & $\mathrm{OM}$ & 1454 & & & & & & & & & & & & & 5.1 & \\
\hline ar-Curcumene & $\mathrm{SH}$ & 1484 & & & & & & & & & & & & & 5.6 & \\
\hline Eugenol acetate & PP & 1529 & & & & & & & & & & & & & & \\
\hline 5-ері-7-ері- $\alpha$-Eudesmol & OS & 1606 & & & & & & & & & & & & & 5.5 & \\
\hline$\gamma$-Eudesmol & OS & 1634 & & & & & & & & & & & & & 0.5 & \\
\hline T-Cadinol & OS & 1642 & & & & & & & & & & & & & & \\
\hline$\beta$-Eudesmol & OS & 1649 & & & & & & & & & & & & & 0.2 & \\
\hline Valerianol & OS & 1655 & & & & & & & & & & & & & 0.3 & \\
\hline 7-epi- $\alpha$-Eudesmol & OS & 1664 & & & & & & & & & & & & & & \\
\hline (Z)- $\alpha$-Santalol & OS & 1675 & & & & & & & & & & & & & & \\
\hline (Z)- $\beta$-trans-Santalol & OS & 1710 & & & & & & & & & & & & & & \\
\hline Unknown & & & 7.9 & 2.8 & 5.1 & 0.3 & & & & 0.8 & & 6.2 & 2.4 & 0.3 & 5.8 & \\
\hline TOTAL & & & 92.1 & 97.2 & 94.9 & 99.7 & 100.0 & 100.0 & 100.0 & 99.2 & 100.0 & 93.9 & 97.6 & 99.7 & 94.3 & 100.0 \\
\hline
\end{tabular}

* Single compounds that showed a percentage less than $5 \%$ in at least one of the tested EOs are removed from this table. \$ Linear retention index. A.n (Anthemis nobilis); A.g (Anethum graveolens); B.s (Boswellia sacra); C.z (Cynnamomum zeylanicum); C.a (Citrus aurantium); C.b (Citrus bergamia); C.l (Citrus limon); C.p (Citrus paradisi); C.a.dul (Citrus aurantium var. dulcis); C.la (Cistus ladanifer); C.c (Cymbopogon citratus); F.v (Foeniculum vulgare); H.i (Helichrysum italicum); I.v (Illicium verum). MH (Monoterpene Hydrocarbons), OM (Oxygenated Monoterpenes), SH (Sesquiterpene Hydrocarbons), OS (Oxygenated Sesquiterpenes), DH (Diterpene Hydrocarbons), OD (Oxygenated Diterpenes), PP (Phenylpropanoids), NT (Non Terpenes), EST (Esters). 
Table 1. Cont.

(b)

\begin{tabular}{|c|c|c|c|c|c|c|c|c|c|c|c|c|c|c|c|c|c|c|}
\hline Compounds * & Class & LRI $\S$ & L.h & L.c & M.c & $O . b$ & O.m & $O . v$ & $P . g$ & P.ab & $P . a$ & R.o & S.s & S.al & S.m & $S . a$ & T.c & $T . v$ \\
\hline Propyl butanoate & EST & 898 & & & & & & & & & & & & & & & & \\
\hline$\alpha$-Thujene & MH & 932 & & & 0.6 & & 0.8 & 0.8 & & 0.5 & & 0.2 & & & 0.3 & & & 0.1 \\
\hline Tricyclene & MH & 938 & 0.5 & 1.5 & 49.0 & & & & & & & 0.2 & & & & & & \\
\hline$\alpha$-Pinene & $\mathrm{MH}$ & 940 & & & & 0.2 & 0.7 & 1.0 & & 10.8 & 0.1 & 37.9 & & & 0.5 & & & 0.9 \\
\hline$\alpha$-Fenchene & MH & 951 & & & & & & 0.1 & & & & & & & & & & \\
\hline Camphene & MH & 955 & 0.5 & 0.3 & 0.2 & 0.1 & & & & 4.6 & & 5.4 & & & 0.3 & & & 0.3 \\
\hline Thuja-2.4(10)-diene & MH & 959 & & & & & & & & & & & & & & & & \\
\hline$\beta$-Pinene & $\mathrm{MH}$ & 981 & 0.4 & 1.2 & 0.9 & 0.5 & 0.7 & 0.4 & & 1.5 & & 5.0 & & & 0.9 & & & \\
\hline$\alpha$-Terpinene & MH & 1019 & & & & & 4.7 & 2.1 & & & & 0.3 & & & 1.2 & & 0.3 & 0.8 \\
\hline p-Cymene & MH & 1028 & & 0.2 & 2.7 & & 4.2 & 9.3 & & & & & & & 9.0 & & 2.4 & 15.3 \\
\hline Limonene & MH & 1032 & & 16.3 & 5.9 & 0.3 & 2.1 & 0.7 & & 20.4 & & 3.3 & & & & & & 0.4 \\
\hline$\beta$-Phellandrene & MH & 1033 & & & & & & & & & & & & & & & & \\
\hline 1.8-Cineole & OM & 1036 & 7.7 & 2.3 & 29.0 & 5.9 & 0.1 & 0.8 & & & & 22.0 & & & 1.0 & & 1.3 & 0.7 \\
\hline Isobutyl angelate & EST & 1053 & & & & & & & & & & & & & & & & \\
\hline$\gamma$-Terpinene & MH & 1062 & 0.1 & 0.1 & & & 7.9 & 5.3 & & & & 0.5 & & & 6.1 & & 1.6 & 2.9 \\
\hline Artemisia ketone & MH & 1065 & & & & & & & & & & & & & & & & \\
\hline Fenchone & $\mathrm{OM}$ & 1090 & & & & & & & & & & & & & & & & \\
\hline trans-Sabinene hydrate & $\mathrm{OM}$ & 1101 & & & & & 12.8 & 1.8 & & & & & & & & & & 3.8 \\
\hline Linalool & $\mathrm{OM}$ & 1102 & 31.5 & 1.5 & 1.5 & 46.0 & & & 3.9 & & 3.5 & 0.6 & 8.1 & & 3.1 & & 2.3 & \\
\hline Camphor & $\mathrm{OM}$ & 1148 & 7.3 & & & 0.8 & 0.2 & & & & & 7.6 & & & 0.7 & & & 0.5 \\
\hline Isoamyl angelate & EST & 1162 & & & & & & & & & & & & & & & & \\
\hline Propyl tiglate & EST & 1166 & & & & & & & & & & & & & & & & \\
\hline 4-Terpineol & $\mathrm{OM}$ & 1180 & 4.0 & 0.1 & 0.2 & 0.3 & 17.6 & 0.9 & & & & 0.3 & & & 0.7 & & 1.7 & 2.4 \\
\hline 3.9-Epoxy-p-menth-1-ene & $\mathrm{OM}$ & 1186 & & & & & & & & & & & & & & & & \\
\hline Menthyl chavicol & PP & 1198 & & & & 1.1 & & & & & 0.4 & & & & & & & \\
\hline Citronellol & $\mathrm{OM}$ & 1231 & & & & & & & 44.5 & & & & & & & & & \\
\hline Neral & $\mathrm{OM}$ & 1242 & & 32.5 & & & & & 0.2 & & & & & & & & & \\
\hline Carvone & $\mathrm{OM}$ & 1248 & & & & & 0.6 & & & 0.8 & & & & & 1.9 & & & \\
\hline Geraniol & $\mathrm{OM}$ & 1259 & & 0.5 & & 0.2 & 2.7 & & 13.7 & & & & & & & & & \\
\hline Linalyl acetate & $\mathrm{OM}$ & 1260 & 26.8 & & & & 3.2 & & & & & & 54.7 & & 1.2 & & & \\
\hline (E)-Cinnamaldehyde & NT & 1274 & & & & & & & & & & & & & & & & \\
\hline Geranial & $\mathrm{OM}$ & 1276 & & 36.4 & & & & & 0.7 & & & & & & & & & \\
\hline Citronellyl formate & $\mathrm{OM}$ & 1280 & & & & & & & 7.3 & & & & & & & & & \\
\hline iso-Bornyl acetate & $\mathrm{OM}$ & 1287 & & & & 1.6 & 0.2 & 0.1 & & 8.9 & & 3.3 & 0.1 & & & & & \\
\hline (E)-Anethol & PP & 1290 & & & & & & & & & 94.6 & & & & & & & \\
\hline Carvacrol & $\mathrm{OM}$ & 1301 & & & & & 20.8 & 65.9 & & & & & & & 47.1 & & 82.5 & 0.2 \\
\hline Thymol & $\mathrm{OM}$ & 1307 & & & & & 0.2 & 0.9 & & & & & & & 2.6 & & & 52.6 \\
\hline
\end{tabular}


Table 1. Cont.

\begin{tabular}{|c|c|c|c|c|c|c|c|c|c|c|c|c|c|c|c|c|c|c|}
\hline Compounds * & Class & LRI ${ }^{\S}$ & L.h & L.c & M.c & O.b & $O . m$ & O.v & $P . g$ & P.ab & P.a & R.o & S.s & S.al & S.m & S. $a$ & T.c & T.v \\
\hline$\alpha$-Limonene diepoxide & OM & 1347 & & & & & & & & & & & 8.6 & & & & & \\
\hline Eugenol & PP & 1361 & & & & 11.5 & & & & & & & & & & 77.9 & & \\
\hline Neryl acetate & $\mathrm{OM}$ & 1368 & 0.4 & & & & & & & & & & 0.1 & & & & & \\
\hline$\beta$-Caryophyllene & SH & 1418 & 2.2 & 0.8 & 0.5 & 0.3 & 1.7 & 3.7 & 0.7 & 4.4 & & 4.1 & & & 3.6 & 8.9 & 3.0 & 6.8 \\
\hline Neryl propanoate & $\mathrm{OM}$ & 1454 & & & & & & & & & & & & & & & & \\
\hline ar-Curcumene & SH & 1484 & & & & & & & & & & & & & 0.3 & & & \\
\hline Eugenol acetate & PP & 1529 & & & & & & & & & & & & & & 12.2 & & \\
\hline 5-epi-7-ерi- $\alpha$-Eudesmol & OS & 1606 & & & & & & & & & & & & & & & & \\
\hline$\gamma$-Eudesmol & OS & 1634 & & & & & & & & & & & & 5.2 & & & & \\
\hline T-Cadinol & OS & 1642 & 0.2 & & & 5.8 & & & & & & & & & 0.2 & & & \\
\hline$\beta$-Eudesmol & OS & 1649 & & & & & & & & & & & & 5.5 & & & & \\
\hline Valerianol & OS & 1655 & & & & & & & & & & & & 14.4 & & & & \\
\hline 7-epi- $\alpha$-Eudesmol & OS & 1664 & & & & & & & & & & & & 5.9 & & & & \\
\hline (Z)- $\alpha$-Santalol & OS & 1675 & & & & & & & & & & & & 27.1 & & & & \\
\hline (Z)- $\beta$-trans-Santalol & OS & 1710 & & & & & & & & & & & & 10.8 & & & & \\
\hline Unknown & & & 6.2 & 0.8 & 2.0 & 1.8 & 0.0 & 1.7 & 1.4 & 5.1 & 13.9 & 5.6 & 5.4 & & 1.3 & 1.7 & & \\
\hline TOTAL & & & 100.0 & 99.4 & 93.9 & 99.2 & 98.1 & 98.2 & 99.3 & 98.3 & 98.6 & 94.9 & 86.2 & 94.4 & 94.6 & 100.0 & 98.7 & 97.3 \\
\hline
\end{tabular}

Single compounds that showed a percentage of less than $5 \%$ in at least one of the tested EOs are removed from this table. \& Linear retention index. L.h (Lavandula hybrida); L.c (Litsea cubeba); M.c (Myrtus communis); O.b (Ocimum basilicum); O.m (Origanum majorana); O.v (Origanum vulgare); P.g (Pelargonium graveolens); P.ab (Picea abies); P.a (Pimpinella anisum); R.o (Rosmarinus officinalis); S.s (Salvia sclarea); S.al (Santalum album); S.m (Satureja montana); S.a (Syzygium aromaticum); T.c (Thymus capitatus); T.v (Thymus vulgaris). MH (Monoterpene Hydrocarbons), OM (Oxygenated Monoterpenes), SH (Sesquiterpene Hydrocarbons), OS (Oxygenated Sesquiterpenes), DH (Diterpene Hydrocarbons), OD (Oxygenated Diterpenes), PP (Phenylpropanoids), NT (Non Terpenes), EST (Esters). 
Table 2. (a) Classes of components present in the first list of tested EOs (1-14); (b) Class of components present in the second list of tested EOs (15-30).

(a)

\begin{tabular}{|c|c|c|c|c|c|c|c|c|c|c|c|c|c|c|}
\hline Class of Compounds * & A.n & A.g & B.s & C.z & C. $a$ & C.b & C.l & C.p & C.a.dul & C.la & C.c & F.v & H.i & $I . v$ \\
\hline Monoterpene hydrocarbons (MH) & 10.3 & 10.1 & 84.3 & 15.5 & 97.4 & 49.0 & 94.3 & 96.2 & 98.7 & 57.0 & 3.9 & 22.1 & 19.2 & 7.3 \\
\hline Oxygenated monoterpenes (OM) & 5.9 & 85.9 & 6.7 & 7.4 & 1.9 & 48.5 & 3.6 & 0.5 & 0.6 & 16.6 & 86.3 & 21.1 & 40.6 & 0.7 \\
\hline Sesquiterpene hydrocarbons (SH) & 0.2 & & 3.7 & 14.7 & 0.2 & 2.5 & 2.0 & 1.9 & & 11.0 & 4.5 & & 22.5 & 1.1 \\
\hline Oxygenated sesquiterpenes (OS) & 0.2 & & & 0.8 & & & & & & 6.6 & 0.9 & & 8.9 & 0.1 \\
\hline \multicolumn{15}{|l|}{ Diterpene hydrocarbons (DH) } \\
\hline \multicolumn{15}{|l|}{ Oxygenated diterpenes (OD) } \\
\hline Phenylpropanoids (PP) & & 0.3 & 0.2 & 3.4 & & & & & & & & 56.5 & & 90.8 \\
\hline Non-terpenes (NT) & & 0.9 & & 57.9 & 0.5 & 0.1 & 0.1 & 0.6 & 0.7 & 2.7 & 2.0 & & & \\
\hline Esters (EST) & 75.6 & & & & & & & & & & & & 3.1 & \\
\hline TOTAL Identified & 92.1 & 97.2 & 94.9 & 99.7 & 100.0 & 100.1 & 100.0 & 99.2 & 100.0 & 93.9 & 97.6 & 99.7 & 94.3 & 100.0 \\
\hline
\end{tabular}

* Each class of compounds represent the total amount (\%) of the identified constituents. A.n (Anthemis nobilis); A.g (Anethum graveolens); B.s (Boswellia sacra); C.z (Cynnamomum zeylanicum); C.a (Citrus aurantium); C.b (Citrus bergamia); C.l (Citrus limon); C.p (Citrus paradisi); C.a.dul (Citrus aurantium var. dulcis); C.la (Cistus ladanifer); C.c (Cymbopogon citratus); F.v (Foeniculum vulgare); H.i (Helichrysum italicum); I.v (Illicium verum).

(b)

\begin{tabular}{|c|c|c|c|c|c|c|c|c|c|c|c|c|c|c|c|c|}
\hline Class of Compounds * & L.h & L.c & M.c & O.b & $O . m$ & O.v & $P . g$ & P.ab & P. $a$ & R.o & S.s & S.al & S.m & S. $a$ & T.c & $T . v$ \\
\hline Monoterpene hydrocarbons (MH) & 6.4 & 21.3 & 61.2 & 2.3 & 27.7 & 22.4 & & 38.2 & 0.1 & 56.5 & & & 19.6 & & 4.6 & 21.5 \\
\hline Oxygenated monoterpenes (OM) & 85.0 & 75.7 & 31.1 & 56.1 & 66.6 & 71.2 & 83.4 & 23.1 & 3.5 & 36.8 & 78.2 & 0.6 & 61.9 & & 89.2 & 64.1 \\
\hline Sesquiterpene hydrocarbons (SH) & 5.4 & 0.9 & 1.2 & 20.0 & 3.2 & 4.2 & 7.8 & 25.0 & & 4.4 & 0.9 & 5.1 & 11.9 & 9.5 & 3.8 & 9.2 \\
\hline Oxygenated sesquiterpenes (OS) & 1.3 & & & 7.9 & 0.4 & 0.4 & 6.9 & 12.0 & & 0.3 & 4.9 & 88.8 & 1.1 & 0.4 & 0.7 & \\
\hline Diterpene hydrocarbons (DH) & & & & & & & & & & & & & & & 0.2 & \\
\hline Oxygenated diterpenes (OD) & & & & & & & & & & & 1.3 & & & & & \\
\hline Phenylpropanoids (PP) & & & & 12.7 & & & 1.2 & & 95.0 & & & & & 90.1 & & \\
\hline Non-terpenes (NT) & 0.8 & 1.5 & & & 0.1 & & & & & & 0.2 & & & & 0.3 & 0.8 \\
\hline Esters (EST) & 1.1 & & 0.3 & 0.2 & & & & & & & & & & & & 1.7 \\
\hline TOTAL Identified & 100.0 & 99.4 & 93.9 & 99.2 & 98.1 & 98.2 & 99.3 & 98.3 & 98.6 & 94.9 & 86.2 & 94.4 & 94.6 & 100.0 & 98.7 & 97.3 \\
\hline
\end{tabular}

* Each class of compounds represent the total amount (\%) of the identified constituents. L.h (Lavandula hybrida); L.c (Litsea cubeba); M.c (Myrtus communis); O.b (Ocimum basilicum);

O.m (Origanum majorana); O.v (Origanum vulgare); P.g (Pelargonium graveolens); P.ab (Picea abies); P.a (Pimpinella anisum); R.o (Rosmarinus officinalis); S.s (Salvia sclarea); S.al (Santalum album); S.m (Satureja montana); S.a (Syzygium aromaticum); T.c (Thymus capitatus); T.v (Thymus vulgaris). 
The microdilution test showed different degrees of inhibition versus the examined Prototheca species. C. paradisi yielded the lowest MIC values $(0.75 \%)$ for both algal species. P. zopfii appeared to be more sensitive to EOs, in comparison to P. blaschkeae. T. vulgaris, L. cubeba, and O. vulgare were effective at $0.75 \%$ versus $P$. zopfii, and at $1 \%$ versus P. blaschkeae. More detailed data are reported in Table 3.

Table 3. Minimal inhibitory concentration (MIC) of tested EOs and conventional antifungal drugs.

\begin{tabular}{|c|c|c|}
\hline EOs & Prototheca zopfii & Prototheca blaschkeae \\
\hline & \multicolumn{2}{|c|}{ MIC (\%) } \\
\hline Pimpinella anisum & 4 & $>4$ \\
\hline Illicium verum & $>4$ & $>4$ \\
\hline Santalum album & $>4$ & $>4$ \\
\hline Helichrysum italicum & $>4$ & $>4$ \\
\hline Rosmarinus officinalis & $>4$ & $>4$ \\
\hline Lavandula hybrida & $>4$ & $>4$ \\
\hline Pelargonium graveolens & $>4$ & $>4$ \\
\hline Salvia sclarea & $>4$ & $>4$ \\
\hline Cynnamomum zeylanicum & $>4$ & $>4$ \\
\hline Foeniculum vulgare & $>4$ & $>4$ \\
\hline Syzygium aromaticum & $>4$ & $>4$ \\
\hline Boswellia sacra & $>4$ & $>4$ \\
\hline Anthemis nobilis & $>4$ & $>4$ \\
\hline Citrus paradisi & 0.75 & 0.75 \\
\hline Citrus bergamia & 2 & 0.75 \\
\hline Citrus aurantium & $>4$ & $>4$ \\
\hline Citrus aurantium var. dulcis & $>4$ & $>4$ \\
\hline Citrus limon & $>4$ & $>4$ \\
\hline Cymbopogon citratus & 1 & $>4$ \\
\hline Ocimum basilicum & 1 & $>4$ \\
\hline Origanum majorana & 1 & $>4$ \\
\hline Thymus vulgaris & 0.75 & 1 \\
\hline Litsea cubeba & 0.75 & 1 \\
\hline Origanum vulgare & 0.75 & 1 \\
\hline Satureja montana & 4 & 4 \\
\hline Cistus ladanifer & 4 & 4 \\
\hline Picea abies & 4 & 4 \\
\hline Anethum graveolens & 4 & 4 \\
\hline Thymus capitatus & 4 & 4 \\
\hline Myrtus communis & 4 & 4 \\
\hline Conventional drugs & \multicolumn{2}{|c|}{$\mathrm{MIC}(\mu \mathrm{g} / \mathrm{mL})$} \\
\hline Posaconazole & 0.38 & 0.5 \\
\hline Voriconazole & 6 & 6 \\
\hline Amphotericin B & 0.25 & 0.19 \\
\hline
\end{tabular}

Both species were susceptible to amphotericin B and posaconazole, while not sensitive to voriconazole.

\section{Discussion}

The present study is the first to investigate the in vitro susceptibility of P. zopfii and P. blaschkeae to a wide range of EOs, obtained from different botanical families.

C. paradisi appeared to possess the most effective EO against both algal species. The activity of this EO against Prototheca spp. had not previously been investigated, and for this reason, our results cannot be compared to already published data.

However, according to comparable results referring to P. zopfii's susceptibility to EOs, MIC values obtained from our microdilution assay seemed to be higher, when compared to those already recorded. In fact, Tortorano et al. [15] reported MICs ranging from $0.03 \%$ to $0.12 \%$ for Melaleuca alternifolia, while MIC values for $C$. bergamia were in agreement with data obtained in the present study. 
Grzesiak et al. [26] described C. zeylanicum EO as the most effective against $P$. zopfii, followed by T. vulgaris, S. aromaticum, and S. sclarea, with MICs ranging from $0.02 \%$ to $0.25 \%$. However, these authors performed the assay following a different method than that implemented in the present study and by Tortorano et al. [15], making a comparison among the different results obtained difficult.

As indicated, T. vulgaris, L. cubeba, and O. vulgare exhibited an inhibitory effect at the level of $0.75 \%$ and $1 \%$ against $P$. zopfii and P. blaschkeae, respectively.

The main component of $C$. paradisi is limonene. Monoterpenes such as limonene and thymol can exert damage on membrane-embedded enzymes, modify fatty acid composition [33], and affect the respiration and permeability of cell membranes [34]. Furthermore, these monoterpenes are inhibitors of pectin methyl esterase and cellulase, causing consequent damage to fungal cell walls [35]. Limonene, in particular, has also been proven to alter the structure and function of the cell wall in Saccharomyces cerevisiae [36].

The activity of some of the examined EOs seem interesting, considering that no drug is currently licensed for use in bovine protothecosis. These compounds could be evaluated for topical administration in a dense excipient for an endomammary treatment of affected cows. The MIC values obtained for the above-mentioned EOs may be employed to treat mammary glands without damaging epithelial cells. Moreover, EOs could be used as disinfectants during milk processing by ensuring a high hygiene level. Protothecal mastitis can in fact be a consequence of poor hygienic conditions, and the search for effective products against these algae is on the rise. The efficacy of some teat disinfectants containing iodine, quaternary ammonium compounds, and dodecylbenzene sulphonic acid has been reported [37], indicating iodine as the most suitable disinfectant. Guanidine is a further compound which is effective both as an antiseptic for human wounds and as a surface disinfectant yielding algicidal activity at low concentration on P. zopfii [8]. More recently, the in vitro effect of iodopropynyl butylcarbamate alone and in combination with amphotericin B was assessed on P. zopfii and P. blaschkeae isolates obtained from dairy herds of different European countries, showing a satisfactory anti-Prototheca activity [38].

All of the above-mentioned products are proven to be active versus $P$. zopfii, but to the best of our knowledge only Jagielski et al. [38] provided information on P. blaschkeae susceptibility. Although not frequently, this species is also reported as responsible for bovine mastitis outbreaks [39].

Further investigations are needed to evaluate the efficacy of EO-based formulations in the disinfection of both udder and milking products.

\section{Conclusions}

Some among the examined EOs-in particular C. paradisi EO-showed interesting antialgal activity against both $P$. zopfii and P. blaschkeae. This finding could contribute to broaden the informational outlook on the unconventional treatment of both mastitic cows and dairy environments.

Author Contributions: Simona Nardoni, Francesca Pisseri, and Francesca Mancianti conceived and designed the experiments; Simona Nardoni performed the experiments and analyzed the data; Basma Najar and Luisa Pistelli analyzed EO composition and performed the statistical analysis; Mario Luini contributed Prototheca strains; and Francesca Mancianti and Simona Nardoni wrote the paper.

Conflicts of Interest: The authors declare no conflict of interest.

\section{References}

1. Roesler, U.; Möller, A.; Hensel, A.; Baumann, D.; Truyen, U. Diversity within the current algal species Prototheca zopfii: A proposal for two Prototheca zopfii genotypes and description of a novel species, Prototheca blaschkeae sp. nov. Int. J. Syst. Evol. Microbiol. 2006, 56, 1419-1425. [CrossRef] [PubMed]

2. Satoh, K.; Ooe, K.; Nagayama, H.; Makimura, K. Prototheca cutis sp. nov., a newly discovered pathogen of protothecosis isolated from inflamed human skin. Int. J. Syst. Evol. Microbiol. 2010, 60, 1236-1240. [CrossRef] [PubMed] 
3. Marques, S.; Huss, V.A.R.; Pfisterer, K.; Grosse, C.; Thompson, G. Internal transcribed spacer sequence-based rapid molecular identification of Prototheca zopfii and Prototheca blaschkeae directly from milk of infected cows. J. Dairy Sci. 2015, 98, 3001-3009. [CrossRef] [PubMed]

4. Masuda, M.; Hirose, N.; Ishikawa, T.; Ikawa, Y.; Nishimura, K. Prototheca miyajii sp. nov., isolated from a patient with systemic protothecosis. Int. J. Syst. Evol. Microbiol. 2016, 66, 1510-1520. [CrossRef] [PubMed]

5. Roesler, U.; Hensel, A. Longitudinal analysis of Prototheca zopfii-specific immune responses: Correlation with disease progression and carriage in dairy cows. J. Clin. Microbiol. 2003, 41, 1181-1186. [CrossRef] [PubMed]

6. Gonçalves, J.L.; Lee, S.H.I.; de Paula Arruda, E.; Galles, D.P.; Caetano, V.C.; de Oliveira, C.A.F.; Fernandes, A.M.; dos Santos, M.V. Biofilm-producing ability and efficiency of sanitizing agents against Prototheca zopfii isolates from bovine subclinical mastitis. J. Dairy Sci. 2015, 98, 3613-3621. [CrossRef] [PubMed]

7. Kwiecinski, J. Biofilm formation by pathogenic Prototheca algae. Lett. Appl. Microbiol. 2015, 61, 511-517. [CrossRef] [PubMed]

8. Alves, A.C.; Capra, E.; Morandi, S.; Cremonesi, P.; Pantoja, J.C.F.; Langoni, H.; Vargas, A.P.C.; Costa, M.M.; Jagielski, T.; Bolaños, C.A.D.; et al. In vitro algicidal effect of guanidine on Prototheca zopfii genotype 2 strains isolated from clinical and subclinical bovine mastitis. Lett. Appl. Microbiol. 2017, 64, 419-423. [CrossRef] [PubMed]

9. Jánosi, S.; Ratz, F.; Szigeti, G.; Kulcsar, M.; Kerenyi, J.; Lauko, T.; Katona, F.; Huszenicza, G. Pathophysiology: Review of the microbiological, pathological, and clinical aspects of bovine mastitis caused by the alga Prototheca zopfii. Vet. Q. 2001, 23, 58-61. [CrossRef] [PubMed]

10. Marques, S.; Silva, E.; Kraft, C.; Carvalheira, J.; Videira, A.; Huss, V.A.; Thompson, G. Bovine mastitis associated with Prototheca blaschkeae. J. Clin. Microbiol. 2008, 46, 1941-1945. [CrossRef] [PubMed]

11. Ahrholdt, J.; Murugaiyan, J.; Straubinger, R.K.; Jagielski, T.; Roesler, U. Epidemiological analysis of worldwide bovine, canine and human clinical Prototheca isolates by PCR genotyping and MALDI-TOF mass spectrometry proteomic phenotyping. Med. Mycol. 2012, 50, 234-243. [CrossRef] [PubMed]

12. Lass-Flörl, C.; Mayr, A. Human protothecosis. Clin. Microbiol. Rev. 2007, 20, 230-242. [CrossRef] [PubMed]

13. Takano, M.; Hoshi, S.; Nagai, K.; Ishidaira, H.; Onozaki, M.; Satoh, K.; Makimura, K. The first case of human protothecosis caused by Prototheca zopfii in Japan. J. Inf. Chemother. 2014, 20, 647-649. [CrossRef] [PubMed]

14. Buzzini, P.; Turchetti, B.; Facelli, R.; Baudino, R.; Cavarero, F.; Mattalia, L.; Mosso, P.; Martini, A. First large-scale isolation of Prototheca zopfii from milk produced by dairy herds in Italy. Mycopathologia 2004, 158, 427-430. [CrossRef] [PubMed]

15. Tortorano, A.M.; Prigitano, A.; Dho, G.; Piccinini, R.; Dapra, V.; Viviani, M.A. In vitro activity of conventional antifungal drugs and natural essences against the yeast-like alga Prototheca. J. Antimicrob. Chemother. 2008, 61, 1312-1314. [CrossRef] [PubMed]

16. Jagielski, T.; Buzzini, P.; Lassa, H.; Malinowski, E.; Branda, E.; Turchetti, B.; Polleichtner, A.; Lagneau, P.E.; Silva, E.; Marques, S.; et al. Multicentre Etest evaluation of in vitro activity of conventional antifungal drugs against European bovine mastitis Prototheca spp. isolates. J. Antimicrob. Chemother. 2012, 67, 1945-1947. [CrossRef] [PubMed]

17. Buzzini, P.; Pieroni, A. Antimicrobial activity of extracts of Clematis vitalba towards pathogenic yeast and yeast-like microorganisms. Fitoterapia 2003, 74, 397-400. [CrossRef]

18. Turchetti, B.; Pinelli, P.; Buzzini, P.; Romani, A.; Heimler, D.; Franconi, F.; Martini, A. In vitro antimycotic activity of some plant extracts towards yeast and yeast-like strains. Phytother. Res. 2005, 191, 44-49. [CrossRef] [PubMed]

19. Pandey, A.K.; Kumar, P.; Singh, P.; Tripathi, N.N.; Bajpai, V.K. Essential oils: Sources of antimicrobials and food preservatives. Front. Microbiol. 2017, 7, 2161. [CrossRef] [PubMed]

20. Vergis, J.; Gokulakrishnan, P.; Agarwal, R.K.; Kumar, A. Essential oils as natural food antimicrobial agents: A review. Crit. Rev. Food Sci. Nutr. 2015, 55, 1320-1323. [CrossRef] [PubMed]

21. Mahboubi, M. Artemisia sieberi Besser essential oil and treatment of fungal infections. Biomed. Pharmacother. 2017, 89, 1422-1430. [CrossRef] [PubMed]

22. Timbermont, L.; Lanckriet, A.; Dewulf, J.; Nollet, N.; Schwarzer, K.; Haesebrouck, F.; Ducatelle, R.; Van Immerseel, F. Control of Clostridium perfringens-induced necrotic enteritis in broilers by target-released butyric acid, fatty acids and essential oils. Avian Pathol. 2010, 39, 117-121. [CrossRef] [PubMed] 
23. Carrasco, F.R.; Schmidt, G.; Romero, A.L.; Sartoretto, J.L.; Caparroz-Assef, S.M.; Bersani-Amado, C.A.; Cuman, R.K.N. Immunomodulatory activity of Zingiber officinale Roscoe, Salvia officinalis L. and Syzygium aromaticum L. essential oils: Evidence for humor-and cell-mediated responses. J. Pharm. Pharmacol. 2009, 61, 961-967. [CrossRef] [PubMed]

24. Miladi, H.; Zmantar, T.; Kouidhi, B.; Chaabouni, Y.; Mahdouani, K.; Bakhrouf, A.; Chaieb, K. Use of carvacrol, thymol, and eugenol for biofilm eradication and resistance modifying susceptibility of Salmonella enterica serovar Typhimurium strains to nalidixic acid. Microb. Pathog. 2017, 104, 56-63. [CrossRef] [PubMed]

25. Peixoto, L.R.; Rosalen, P.L.; Ferreira, G.L.S.; Freires, I.A.; de Carvalho, F.G.; Castellano, L.R.; de Castro, R.D. Antifungal activity, mode of action and anti-biofilm effects of Laurus nobilis Linnaeus essential oil against Candida spp. Arch. Oral Biol. 2017, 73, 179-185. [CrossRef] [PubMed]

26. Grzesiak, B.; Głowacka, A.; Krukowski, H.; Lisowski, A.; Lassa, H.; Sienkiewicz, M. The in vitro efficacy of essential oils and antifungal drugs against Prototheca zopfii. Mycopathologia 2016, 181, 609-615. [CrossRef] [PubMed]

27. Bouari, C.; Bolfa, P.; Borza, G.; Nadăş, G.; Cătoi, C.; Fiţ, N. Antimicrobial activity of Mentha piperita and Saturenja hortensis in a murine model of cutaneous protothecosis. J. Mycol. Méd. 2014, 24, 34-43. [CrossRef] [PubMed]

28. Pistelli, L.; Najar, B.; Giovanelli, S.; Lorenzini, L.; Tavarini, S.; Angelini, L.G. Agronomic and phytochemical evaluation of lavandin and lavender cultivars ciultivated in the Tyrrhenian area of Tuscany (Italy). Ind. Crops Prod. 2017, 109, 37-44. [CrossRef]

29. Ricchi, M.; Cammi, G.; Garbarino, C.A.; Buzzini, P.; Belletti, G.L.; Arrigoni, N. A rapid real-time PCR/DNA resolution melting method to identify Prototheca species. J. Appl. Microbiol. 2011, 110, 27-34. [CrossRef] [PubMed]

30. Clinical and Laboratory Standards Institute CLSI. Reference Method for Broth Dilution Antifungal Susceptibility Testing of Yeasts, 3rd ed.; Clinical and Laboratory Standards Institute: Wayne, NJ, USA, 2008.

31. Pisseri, F.; Scuola Cimi Koinè Roma, Italy. Personal communication, 2017.

32. Sobukawa, H.; Kano, R.; Ito, T.; Onozaki, M.; Makimura, K.; Hasegawa, A.; Kamata, H. In vitro susceptibility of Prototheca zopfii genotypes 1 and 2. Med. Mycol. 2011, 49, 222-224. [CrossRef] [PubMed]

33. Sikkema, J.; De Bont, J.A.; Poolman, B. Mechanisms of membrane toxicity of hydrocarbons. Microbiol. Rev. 1995, 59, 201-222. [PubMed]

34. Cox, S.D.; Mann, C.M.; Markham, J.L.; Bell, H.C.; Gustafson, J.E.; Warmington, J.R.; Wyllie, S.G. The mode of antimicrobial action of the essential oil of Melaleuca alternifolia (tea tree oil). J. Appl. Microbiol. 2000, 88, 170-175. [CrossRef] [PubMed]

35. Marei, G.I.K.; Rasoul, M.A.A.; Abdelgaleil, S.A. Comparative antifungal activities and biochemical effects of monoterpenes on plant pathogenic fungi. Pest Biochem. Physiol. 2012, 103, 56-61. [CrossRef]

36. Brennan, T.C.; Turner, C.D.; Krömer, J.O.; Nielsen, L.K. Alleviating monoterpene toxicity using a two-phase extractive fermentation for the bioproduction of jet fuel mixtures in Saccharomyces cerevisiae. Biotechnol. Bioeng. 2012, 109, 2513-2522. [CrossRef] [PubMed]

37. Lassa, H.; Jagielski, T.; Malinowski, E. Effect of different heat treatments and disinfectants on the survival of Prototheca zopfii. Mycopathologia 2011, 171, 177-182. [CrossRef] [PubMed]

38. Jagielski, T.; Bakuła, Z.; Di Mauro, S.; Casciari, C.; Cambiotti, V.; Krukowski, H.; Turchetti, B.; Ricchi, M.; Manuali, E.; Buzzini, P. A comparative study of the in vitro activity of iodopropynyl butylcarbamate and amphotericin B against Prototheca spp. isolates from European dairy herds. J. Dairy Sci. 2017, 100, 7435-7445. [CrossRef] [PubMed]

39. Ricchi, M.; De Cicco, C.; Buzzini, P.; Cammi, G.; Arrigoni, N.; Cammi, M.; Garbarino, C. First outbreak of bovine mastitis caused by Prototheca blaschkeae. Vet. Microbiol. 2013, 162, 997-999. [CrossRef] [PubMed]

(C) 2018 by the authors. Licensee MDPI, Basel, Switzerland. This article is an open access article distributed under the terms and conditions of the Creative Commons Attribution (CC BY) license (http:// creativecommons.org/licenses/by/4.0/). 